\title{
Reading order and A-B vs B-A performance*
}

\author{
BARRY J. WILSON \\ University of Missouri, Columbia, Mo. 65201
}

Thirty-two college Ss were presented with a PA task using common words. The LR group received the traditional left-right pairing of $A-B$ terms after prior presentation of $A$. The $R L$ group received A-B terms presented right-to-left, or B-A. However, order of pronunciation was A-B for all Ss, and B was always the term to be recalled from memory during learning trials. Immediate bidirectional tests of recall showed $A-B$ recall to be unaffected by reading order, but B-A recall was significantly depressed by the traditional left-to-right order. A matching test administered at a later date gave support for the reading order effect. Results were discussed both according to an incidental learning model and a feature tagging model.

Studies of A-B and B-A performance on PA lists using the anticipation method have rather consistently given evidence against the Asch \& Ebenholtz (1962) principle of associative symmetry (Ekstrand, 1966). One preexperimental habit which has aroused little attention is that of left-to-right reading order. Newman \& Campbell (1971) investigated reading order and A-B, B-A performance using single digits and a study-test methodology. Their results gave a significant Reading Order by Test Direction interaction with the A-B mean higher than the B-A mean for left-to-right-reading Ss and B-A mean higher for right-to-left-reading Ss. They interpret their results as suggesting that it is the temporal order of responding to the two terms of a pair during pairing trials which is the major factor in determining asymmetry, since performance is better when the item more recently responded to is the one to be given on the test. Typical reading habits may also have been disturbed by Kanak \& Neuner (1970), who used a study-test methodology with high meaningful word pairs in two conditions, which differed as to whether A or B was the to-be-recalled item during test phases. Their results show that when $B$ was the recalled item, A-B recall exceeded $B$-A recall while the reverse was true when $A$ was the recalled item. Results were interpreted in terms of Horowitz, Norman, \& Day's (1966) contention that item availability grows fastest when the $\mathrm{S}$ produces the item from memory.

Both the Newman \& Campbell (1971) and Kanak \& Neuner (1970) studies leave unanswered the question of an effect due to reading order. Such a test is available, using a PA anticipation task where the $\mathbf{A}$ term is presented alone (e.g., DOG), followed by the A and B term paired together. The A-B pair can be presented in the traditional left-to-right reading order (DOG CAT) or in a right-to-left reading order (CAT DOG). In both cases, the order of emission is the same (given

*This manuscript is sponsored by Donald H. Kausler, who takes full editorial responsibility for it. appropriate practice and instruction) and $B$ is always the term to be recalled from memory. Differences in A-B or B-A recall under the two conditions should be attributable to differences in reading order.

\section{METHOD AND DESIGN \\ Subjects}

The Ss were 32 college undergraduate volunteers from an educational psychology course.

\section{Apparatus}

Two identical standard Lafayette memory drums were used. One was used for practice trials, the other for learning trials and paced bidirectional recall tests.

\section{Design}

There were two variations of list presentation, left-to-right (LR) or right-to-left (RL), and two orders of recall testing, forward recall (FR) then backward recall (BR) or BR and then FR, giving a 2 by 2 analysis of variance design.

\section{Procedure}

The list used was identical to that used by Kanak \& Neuner (1970) and consisted of word pairs of high T-L frequency (AA) and minimal associative relations as determined by Palermo \& Jenkins (1964) norms. The two list variants were identical, except that the LR order retained the A-B pairing after prior exposure of $\mathrm{A}$, while the $\mathrm{RL}$ order was presented B-A. Three random serial position orders of the list were employed. All Ss were first given a practice list and instructions relevant to their task. Instructions and practice were identical for each group, with the exception of their reading order. All Ss practiced to a criterion of three consecutive errorless trials at a 4-sec rate, followed by three consecutive errorless trials at a $2-\mathrm{sec}$ rate. Practice items were three pairs of highly related common words (e.g., DOG CAT). No S made any errors beyond the first practice item. Ss pronounced each word as it appeared. LR Ss pronounced from left to right, $\mathrm{RL}$ pronounced from right to left, thus order of emission was constant. Following practice, Ss learned the PA task to a criterion of 6 of 8 correct anticipations, which was immediately followed by forward and backward tests of recall counterbalanced for order. In the forward test, Ss responded to a random list of $A$ items at a 2 -sec rate. In the backward test, Ss responded to B items at a 2 -sec rate.

\section{RESULTS}

Two Ss in the RL condition lapsed into their old reading habits and rehearsed left-to-right during some learning trials. Their results were eliminated. Analysis of variance was performed for unequal Ns, using the unweighted means analysis provided by Winer (1971).

\section{Acquisition}

The mean trials to criterion and their standard deviations were 5.87 and 2.87 for the LR group and 6.85 and 3.41 for the RL group. Comparison between means yielded a nonsignificant $t$ of less than 1 . It is concluded that there are no significant differences in learning rate for the two groups.

Tests of Recall

A-B recall was analyzed with a two-way analysis of 
variance, with reading order and order of recall the independant variables. The LR group recalled 6.00 pairs when order of recall was forward then backward and 7.12 pairs when order of recall was backward then forward. The RL group recalled 6.42 and 7.14, respectively. There was no significant main effect for reading order or order of recall (although the latter approached .10) and no interaction. For B-A recall, the LR group recalled 4.63 and 5.38 pairs for the two orders of recall, while the $\mathrm{RL}$ groups recalled 6.14 and 6.29 pairs, respectively. The reading-order effect was significant, $F(1,26)=4.61, p<.05$, but no effect for order of recall and no interaction were found. However, the homogeneity of variance assumption appeared to be in danger with $F \max =10.41, p=.05$. Therefore, further analysis with the Mann-Whitney $U$ test was conducted. This yielded a value for $U$ of $66.5, p=.029$, which suggests that the populations were not drawn from the same population and leading to rejection of the null at the .05 level.

A further test in the classroom was conducted 10 days after the end of the experimental sessions. All participants were given two sheets of paper with two lined columns on each sheet. On the first sheet, they were instructed to write down all the experimental word pairs they could recall from memory. These sheets were then collected. At that point, a blackboard was exposed containing a matrix of words, including those learned experimentally randomly distributed among other familiar words (one primary associate of each of the experimental words). Ss were then instructed to reconstruct the word pairs as they had learned them. The LR group recalled a total of 22 pairs, 19 in the A-B direction and 3 in the B-A direction. The RL group recalled a total of 37 pairs, with 35 A-B pairs and 2 B-A. On the recognition matching task, the LR group $(N=13)$ reconstructed correctly a total of 56 pairs. The $\mathrm{RL}$ group $(\mathrm{N}=12)$ reconstructed a total of 53 pairs. However, the LR group reconstructed 52 pairs in the A-B direction and 4 in the B-A direction, while the RL group reconstructed 30 A-B pairs and 23 B-A pairs. Comparison of the proportion of pairs reconstructed in the A-B and the B-A direction for the $\mathrm{LR}$ and RL groups yields a significant chi square of $18.25, \mathrm{p}<.001$.

\section{DISCUSSION}

Results suggest that reading order does have a significant effect on B-A recall but is not inconsistent with previous studies where effects were attributed to other factors. It is of interest to note that in spite of the rapid alternation between storage and retrieval processes in the anticipation method. RL Ss were able to process the pairs in a relatively unfamiliar manner without serious detriment to learning rate. This outcome is supported by Monge (1971), who found no reading order effects on rate of learning a PA task. Likewise, A-B recall for the RL group was not impaired, but B-A recall was facilitated relative to the $L R$ group.

If B-A learning is a type of incidental learning, as some have contended, right-to-left reading order may have facilitated covert rehearsal in the B-A direction. This explanation is not unlikely, since reading the pairs from left-to-right could be accomplished prior to pronouncing from right-to-left.

A feature-tagging explanation of the results also has some plausibility. In this view, occurrence of each item is postulated to tag various sensory and semantic features of permanent memory. Perhaps visual features of the presented pairs are extracted and stored along with other features extracted during rehearsal. During practice, a RL S learns to suspend normal reading processes from left-to-right to right-to-left, but this does not affect the sensory image, which is registered and stored along with other extracted features.

An interesting question arises concerning final recall and recognition measures taken in the classroom. Recall measure showed few B-A pairs by either group, while recognition measures show $43 \%$ B-A responses for the RL group. This could be interpreted as supporting a featural interpretation. The recognition test was essentially a matching task with visual cues given. The LR group extracted a visual featural component from permanent storage that was in the A-B direction, while the RL group extracted and retrieved in the B-A direction. Due to the unavailability of contrary featural cues, items were matched according to visual featural cues.

Additional investigation may provide answers as to the reliability of the results, results with samples having acquired less left-to-right bias (children), possible effects of reading order on item availability, and verification of differences in A-B, B-A pairing under recall and recognition measurement conditions.

\section{REFERENCES}

Asch, S. E., \& Ebenholtz, M. The principle of associative symmetry. Proceedings of the American Philosophical Society, 1962, 106, 135-163.

Ekstrand, B. R. Backward associations. Psychological Bulletin, $1966,65,50-64$

Horowitz, L. M., Norman, S. A., \& Day, R. A. Availability and associative symmetry. Psychological Review, 1966, 73, 1-15.

Kanak, N. J., \& Neuner, S. D. Associative symmetry and item availability as a function of five methods of paired associate acquisition. Journal of Experimental Psychology, 1970,86, 288-295.

Newman, S. E., \& Campbell, R. T. A-B and B-A performance as functions of test instructions and reading order. Journal of Ex perimental Psychology, 1971, 88, 57-59.

Monge, R. H. Studies of verbal learning from the college years through middle age. Journal of Gerontology, 1971, 26, 324-329.

Palermo, D. S., \& Jenkins, J. J. Word association norms: Grade school through college. Minneapolis: University of Minnesota Press, 1964.

Winer, B. J. Statistical principles in experimental design. New York: McGraw-Hill, 1971.

(Received for publication A pril 26, 1973.) 